\title{
Do cancer patients with dementia receive less aggressive treatment in end-of-life care? A nationwide population-based cohort study
}

\author{
Huei-Kai Huang1, Jyh-Gang Hsieh ${ }^{1,2}$, Chia-Jung Hsieh ${ }^{3}$ and Ying-Wei Wang ${ }^{1,2}$ \\ ${ }^{1}$ Department of Family Medicine, Buddhist Tzu Chi General Hospital, Hualien, Taiwan \\ ${ }^{2}$ School of Medicine, Tzu Chi University, Hualien, Taiwan \\ ${ }^{3}$ Department of Public Health, Tzu Chi University, Hualien, Taiwan \\ Correspondence to: Ying-Wei Wang, email: drywwang@gmail.com \\ Keywords: cancer, dementia, palliative care, terminal care, end-of-life care \\ Received: January 28, $2017 \quad$ Accepted: June 02, 2017 Published: June 29, 2017 \\ Copyright: Huang et al. This is an open-access article distributed under the terms of the Creative Commons Attribution License \\ 3.0 (CC BY 3.0), which permits unrestricted use, distribution, and reproduction in any medium, provided the original author and \\ source are credited.
}

\section{ABSTRACT}

Dementia is a progressive, incurable disease that can deprive patients of the ability to make decisions. This study determines whether dementia influences the medical care that a cancer patient receives at the end of life. We conducted a nationwide population-based cohort study on patients aged $\geq 20$ with newly diagnosed cancer during 2000-2012. After matching to reduce confounders, there were 7,111 patients with and 28,444 without dementia. The adjusted odd ratios (OR) for medical interventions, including intensive care, palliative care, invasive procedures, and advanced diagnostic testing, were calculated for the final month and three months of life by a multiple logistic regression model. In the final month before death, the dementia cohort had longer hospital stays (17.7 vs. 17.1 days), more intensive care unit stays $(O R=1.32)$, and less palliative care $(O R=0.80)$ than the non-dementia cohort and were more likely to receive invasive procedures, including cardiopulmonary resuscitation $(O R=1.32)$, endotracheal intubation (OR $=1.27)$, mechanical ventilation $(O R=1.45)$, urinary catheterization $(O R=1.24)$, and feeding tube $(O R=1.88)$, but less likely to undergo chemotherapy $(O R=0.60)$ and diagnostic procedures such as computed tomography, magnetic resonance imaging, and sonography $(O R=0.87)$ or bone scan $(O R=0.69)$. The analysis examining the three months before death had similar results. In summary, patients with cancer and dementia are more likely to receive intensive care and invasive procedures but less likely to undergo advanced diagnostic testing, chemotherapy, or hospice care than those with cancer but without dementia.

\section{INTRODUCTION}

Despite the possibility of early detection and substantial improvements in treatment and survival, cancer remains the leading cause of death in most developed countries [1]. In Taiwan, cancer accounted for $28.6 \%$ of all deaths in 2015 [2]. Even though greater importance is being attached to the quality of medical care provided to terminal cancer patients [3] and a growing body of evidence indicates that treatment intensity is not positively correlated with health outcome or satisfaction [4-6], the aggressiveness of end-of-life care in cancer patients is increasing [1,7]. Admission to the intensive care unit and receiving chemotherapy in the end-of-life were considered as aggressive treatments. On the other hand, receiving palliative care program was considered as a less aggressive treatment [8]. Aggressive treatment administered at the end-of-life may confer disproportionately small benefits. 
Moreover, aggressive treatment can pose society-wide economic burdens [9].

Dementia is a progressive, incurable disease that can deprive patients of the ability to make decisions for endof-life care [10]. If the wishes of patients with dementia are unknown, they may be subjected to more invasive intervention at the end of life compared to those without dementia [11]. At present, few studies have investigated the influence of dementia on aggressive end-of-life care, and the conclusions drawn by these studies have been inconsistent. For example, Afzal et al. found that invasive interventions administered to patients with dementia did not differ from those administered to patients without dementia, although the former received hospice care less frequently [12]. However, other studies have reported that patients with dementia receive fewer invasive interventions than other patients $[6,13,14]$.

Although a significant overlap of cancer and dementia can be expected because age is a major risk factor for both conditions $[15,16]$, little research has been focused on the caring of those with both dementia and terminal cancer [6]. However, these issues are important for the specific population because individuals with dementia may have impairment in communicating and understanding that may increase the difficulty in diagnosing or treating cancer and discussing the issues related to death and dying. Patients' family members have to make surrogate decisions for them when they lack the capacity to do so, and this can be distressing for both groups.

To address these research gaps, we used nationwide, population-based data from Taiwan to compare medical care administered to patients with terminal cancer with and without dementia during the final months prior to death.

\section{RESULTS}

\section{Patient characteristics}

We identified 549,827 patients with newly diagnosed colorectal, liver, lung, breast, oral, or prostate cancer from the nationwide cohort between 2000 and 2012. After excluding patients not meeting study criteria $(\mathrm{n}=349,571)$, we matched 7,111 patients with both cancer and dementia to 28,444 patients with cancer but without dementia (Figure 1). Variables, including age, gender, type of primary cancer, and length of time between cancer diagnosis and death, were balanced between the two groups of patients. Insurance premium (as a proxy of income) and Charlson comorbidity index varied slightly between the groups (Table 1).

\section{Medical care utilization}

The 1-month analysis (i.e., analysis of care given within 1 month prior to death) revealed that patients with both cancer and dementia had longer hospital stays than those without dementia (17.7 vs. 17.1 days, $\mathrm{p}<0.0001)$. They also had a higher likelihood of intensive care unit (ICU) admission (OR $=1.32,95 \%$ CI 1.25-1.39) and a lower likelihood of receiving palliative care $(\mathrm{OR}=0.80$, 95\% CI 0.74-0.86), including hospice ward care (OR $=0.85,95 \%$ CI $0.79-0.92)$ and palliative consultation service $(\mathrm{OR}=0.61,95 \%$ CI 0.51-0.72) (Table 2). However, the two groups had no significant difference in terms of the hospice home care they received (OR $=1.05,95 \%$ CI $0.94-1.17$ ). The 3 -month analysis (i.e., the analysis of care given within the 3 months prior to death) showed results similar to the 1-month analysis (Table 2).

\section{Use of invasive procedures and advanced diagnostic testing}

Overall, patients with both cancer and dementia received more invasive procedures but less chemotherapy and advanced diagnostic testing than those without dementia.

In the 1-month analysis, patients in the dementia group had a higher likelihood of receiving invasive procedures, including cardiopulmonary resuscitation $(\mathrm{CPR})(\mathrm{OR}=1.32,95 \% \mathrm{CI} 1.22-1.43)$, endotracheal intubation $(\mathrm{OR}=1.27,95 \% \mathrm{CI} 1.19-1.34)$, mechanical ventilation $(\mathrm{OR}=1.45,95 \% \mathrm{CI} 1.37-1.53)$, urinary catheterization $(\mathrm{OR}=1.24,95 \% \mathrm{CI} 1.18-1.30)$, or a feeding tube $(\mathrm{OR}=1.88,95 \%$ CI 1.76-2.00). However, a smaller proportion of patients with dementia received chemotherapy $(\mathrm{OR}=0.60,95 \%$ CI $0.55-0.66)$. They were also less likely to undergo diagnostic imaging, including computed tomography (CT), magnetic resonance imaging (MRI), or sonography $(\mathrm{OR}=0.87,95 \%$ CI $0.82-0.92)$ and bone scans $(\mathrm{OR}=0.69,95 \%$ CI $0.61-0.77)$. There were no significant differences between the two in terms of panendoscopy, colonoscopy, and positron emission tomography (PET) scans (Table 3).

The 3-month analysis yielded identical patterns to those of the 1-month analysis with regard to invasive procedures. Significantly fewer patients in the dementia group had a CT, MRI, sonography, or a bone scan. In the 3-month analysis, they also underwent significantly fewer panendoscopies $(\mathrm{OR}=0.91,95 \%$ CI $0.85-0.90)$ and PET scans $(\mathrm{OR}=0.61,95 \%$ CI 0.40-0.91) (Table 3).

\section{DISCUSSION}

This nationwide, population-based, retrospective cohort study of patients with terminal cancer found that those with dementia were more likely to be admitted to the ICU and receive invasive procedures, but less likely to be given chemotherapy, receive palliative care, or undergo advanced diagnostic testing than those without dementia.

We found that patients with both cancer and dementia were less likely to receive chemotherapy; this was consistent with results of a previous research [6]. 
A systematic literature review found that elderly patients tend to follow doctors' suggestions when deciding whether to undergo chemotherapy [17]. Moreover, a previous study conducted in Asia observed that performance status and comorbidities are the two main factors that doctors consider when deciding about the administration of chemotherapy [18]. This helps explain why a lower proportion of patients with dementia received chemotherapy than the matched patients without dementia.

Conversely, our findings of a higher likelihood for invasive procedures in patients with dementia are not entirely consistent with those of previous studies [6, 12-14]. There are several possible explanations for the discrepancy.
1. The relationship between culture and medical decisions: Previous research has shown that cultural attitudes toward truth-telling, life-extension, and endof-life decisions vary significantly, particularly between Western and Asian cultures [19-22]. These cultural factors can influence whether patients with dementia undergo more aggressive interventions at the end of life. In countries such as Taiwan, where "family consent for disclosure" and "family autonomy" are prominent, diagnosis and prognosis are often revealed to family members before the patients themselves are told. Therefore, family members may make the medical decisions, even when the patient is completely lucid [23]. A research conducted

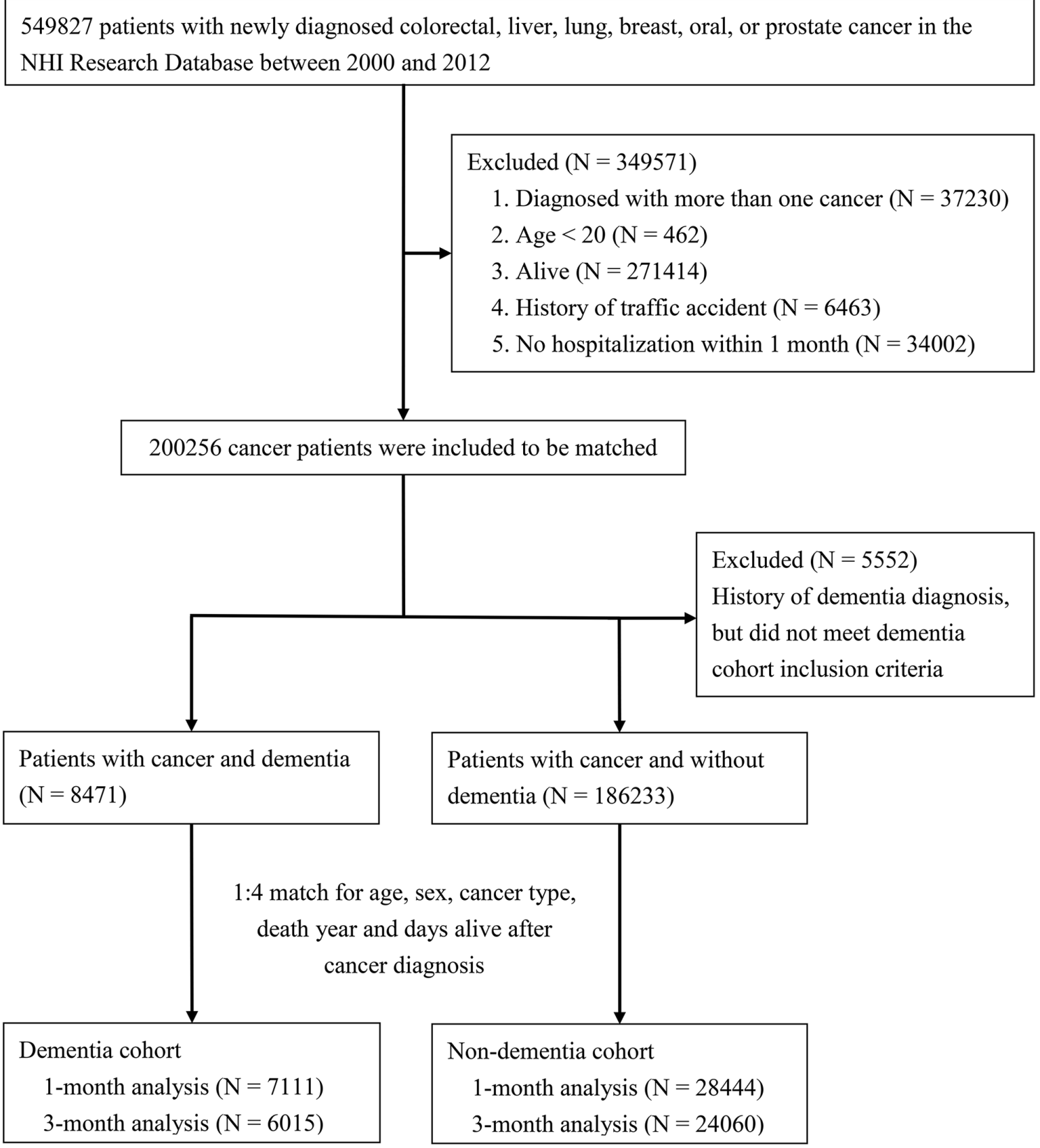

Figure 1: Flow diagram for selection of study subjects. 


\begin{tabular}{|c|c|c|c|c|}
\hline & \multicolumn{2}{|c|}{$\begin{array}{l}\text { Dementia } \\
(\mathrm{N}=7111)\end{array}$} & \multicolumn{2}{|c|}{$\begin{array}{l}\text { Non-dementia } \\
(\mathrm{N}=\mathbf{2 8 4 4 4 )}\end{array}$} \\
\hline & $\mathbf{N}$ & $\%$ & $\mathbf{N}$ & $\%$ \\
\hline \multicolumn{5}{|l|}{ Cancer type } \\
\hline Colorectal cancer & 1942 & 27.3 & 7768 & 27.3 \\
\hline Liver cancer & 1602 & 22.5 & 6408 & 22.5 \\
\hline Lung cancer & 1948 & 27.4 & 7792 & 27.4 \\
\hline Breast cancer & 300 & 4.2 & 1200 & 4.2 \\
\hline Oral cancer & 301 & 4.2 & 1204 & 4.2 \\
\hline Prostate cancer & 1018 & 14.3 & 4072 & 14.3 \\
\hline \multicolumn{5}{|l|}{ Age at death (years) } \\
\hline$<70$ & 590 & 8.3 & 2360 & 8.3 \\
\hline $70-74$ & 782 & 11.0 & 3128 & 11.0 \\
\hline $75-79$ & 1507 & 21.2 & 6028 & 21.2 \\
\hline $80-84$ & 2003 & 28.2 & 8012 & 28.2 \\
\hline$\geq 85$ & 2229 & 31.4 & 8916 & 31.4 \\
\hline \multicolumn{5}{|l|}{ Gender } \\
\hline Male & 4521 & 63.6 & 18084 & 63.6 \\
\hline Female & 2590 & 36.4 & 10360 & 36.4 \\
\hline \multicolumn{5}{|l|}{ Year of death } \\
\hline $2000-2003$ & 349 & 4.9 & 1396 & 4.9 \\
\hline 2004-2007 & 1865 & 26.2 & 7460 & 26.2 \\
\hline 2008-2012 & 4897 & 68.9 & 19588 & 68.9 \\
\hline Cancer diagnosis to death* (years) & 2.19 & 2.53 & 2.02 & 2.33 \\
\hline \multicolumn{5}{|l|}{ Insurance premium (NTD) } \\
\hline Financially dependent & 2096 & 29.5 & 8215 & 28.9 \\
\hline $1-15840$ & 1557 & 21.9 & 5496 & 19.3 \\
\hline $15841-25000$ & 2937 & 41.3 & 12636 & 44.4 \\
\hline 25001 & 521 & 7.3 & 2095 & 7.4 \\
\hline \multicolumn{5}{|l|}{ Charlson comorbidity index } \\
\hline $0-3$ & 1759 & 24.7 & 11822 & 41.6 \\
\hline $4-6$ & 3545 & 49.9 & 10321 & 36.3 \\
\hline $7-9$ & 1189 & 16.7 & 4368 & 15.4 \\
\hline$\geq 10$ & 618 & 8.7 & 1933 & 6.8 \\
\hline
\end{tabular}

*Mean and SD

NTD, New Taiwan dollars

in Taiwan found that end-of-life care preferences of patients with cancer and their family caregivers may differ more than they do in Western countries. Family members may be more accepting of aggressive care and assertive in requesting it than are patients themselves when it comes to invasive and life-support measures [21]. 
Table 2: Comparison of utilization of medical care and palliative care service between cancer patients with and without dementia 1 and 3 months prior to death

\begin{tabular}{|c|c|c|c|c|c|c|c|c|c|c|}
\hline & \multicolumn{5}{|c|}{1 month prior to death } & \multicolumn{5}{|c|}{3 months prior to death } \\
\hline & \multicolumn{2}{|c|}{$\begin{array}{l}\text { Dementia } \\
(N=7111)\end{array}$} & \multicolumn{2}{|c|}{$\begin{array}{l}\text { Non-dementia } \\
(\mathrm{N}=\mathbf{2 8 4 4 4})\end{array}$} & \multirow[b]{2}{*}{$\begin{array}{c}\text { Adjusted OR } \\
(95 \% \text { CI }) \dagger\end{array}$} & \multicolumn{2}{|c|}{$\begin{array}{c}\text { Dementia } \\
(N=6015)\end{array}$} & \multicolumn{2}{|c|}{$\begin{array}{c}\text { Non- } \\
\text { dementia } \\
(\mathrm{N}=\mathbf{2 4 0 6 0 )}\end{array}$} & \multirow[b]{2}{*}{$\begin{array}{c}\text { Adjusted OR } \\
(95 \% \mathrm{CI}) \dagger\end{array}$} \\
\hline & $\mathbf{N}$ & $\%$ & $\mathbf{N}$ & $\%$ & & $\mathbf{N}$ & $\%$ & $\mathbf{N}$ & $\%$ & \\
\hline Inpatient day $\$$ (days, Mean \pm SD) & \multicolumn{2}{|c|}{$17.7 \pm 10.3$} & \multicolumn{2}{|c|}{$17.1 \pm 10.1$} & $\mathrm{p}<0.0001 * * *$ & \multicolumn{2}{|c|}{$33.8 \pm 26.4$} & \multicolumn{2}{|c|}{$30.2 \pm 24.0$} & $\mathrm{p}<0.0001 * * *$ \\
\hline Intensive care & 2768 & 38.9 & 9192 & 32.3 & $1.32(1.25-1.39)^{* * *}$ & 2668 & 44.4 & 8628 & 35.9 & $1.39(1.31-1.48) * * *$ \\
\hline Palliative care & 1142 & 16.1 & 5529 & 19.4 & $0.80(0.74-0.86)^{* * *}$ & 966 & 16.1 & 4807 & 20.0 & $0.77(0.71-0.83) * * *$ \\
\hline Hospice ward care & 1014 & 14.3 & 4683 & 16.5 & $0.85(0.79-0.92)^{* * *}$ & 857 & 14.3 & 4002 & 16.6 & $0.84(0.78-0.91) * * *$ \\
\hline Palliative consultation service & 180 & 2.5 & 1131 & 4.0 & $0.61(0.51-0.72)^{* * *}$ & 172 & 2.9 & 1122 & 4.7 & $0.58(0.49-0.69)^{* * *}$ \\
\hline Hospice home care & 463 & 6.5 & 1776 & 6.2 & $1.05(0.94-1.17)$ & 473 & 7.9 & 1823 & 7.6 & $1.05(0.95-1.17)$ \\
\hline
\end{tabular}

$\dagger$ Multiple logistic regression with adjusted for age, sex, primary malignancy, Charlson comorbidity index, year of death, and insurance premium.

$\$ \mathrm{P}$ values for differences in means determined by independent $\mathrm{t}$ test

$* \mathrm{p}<0.05, * * \mathrm{p}<0.01, * * * \mathrm{p}<0.0001$

Another study found that family members tend to want patients with dementia to receive invasive procedure near the end of their lives, but they would refuse such treatment for themselves under similar circumstances [24]. Since families tend to dominate medical decision making even for competent patients, it is especially true for patients with dementia who cannot clearly express their own wishes. Moreover, eastern culture is a highcontext culture. In other words, people tend to address issues such as illness less directly and explicitly than Westerners. Therefore, in emergency situations, doctors often ask family members to decide whether invasive procedures such as CPR or endotracheal intubation should be administered. However, this action is not consistent with the habits in Eastern, high-context culture, which can influence the decisions of family members [25]. A previous study in terminally ill Taiwanese cancer patients showed that the patient's awareness of prognosis, patientfamily caregiver congruence on the preferred place of death, and the subjective family caregiving burden had a significant impact on the quality of life [26]. This gives further prominence to the importance of the role of family caregiver in Asian cultures such as in Taiwan.

2. Lack of advance directives: Advance directives are rarer in Taiwan than in other countries [27, 28]. In traditional Chinese culture, people are less willing to talk about death. Indeed, talking about death with elderly individuals is taboo because it is believed to bring bad fortune. Even with the constant promotion of hospice care in recent years, children are expected to provide their parents with high-intensity treatment unless the parents themselves direct otherwise. If the family members do not insist on aggressive treatment, others may see them as having given up on their parents or lacking in filial piety $[29,30]$. Therefore, in the absence of advance directives, family members of patients with dementia can have difficulty accepting hospice care or reducing the number of invasive interventions, even if a doctor suggests that doing so is the best course of action.

3. Influence of National Health Insurance (NHI): The Taiwanese healthcare system features good accessibility, comprehensive coverage, short waiting times, and relatively low cost [31]. Almost all medical treatments are covered under the NHI program. Moreover, all patients with cancer can apply for a catastrophic illness certificate. Patients with this certification can access medical care without copayments [32]. As a result, cost is not a deterrent to accepting more aggressive treatment as neither the patient nor the family has to pay any additional fees to receive it.

The results of this study also suggest that patients with both cancer and dementia tend to undergo fewer advanced diagnostic procedures, a factor not examined in other studies. Our finding may be explained by the fact that unlike emergent invasive interventions, such as endotracheal intubation or CPR, doctors order diagnostic studies at their own discretion and discussion with family members. Therefore, if a patient with dementia is near the end of life, the doctor may decide there is no benefit to the patient in pursuing further diagnostic procedures and will not even suggest it. Family members tend to accept this decision without seeing it as having an impact on their view of filial piety. Finally, practical difficulties can also play a role in decisions pertaining to advanced testing. For example, dementia or behavioral and spatial temporal disorders can make performing procedures such as MRIs, scans, or panendoscopy more difficult, changing the riskbenefit and cost-benefit ratios in such cases [6].

The primary strength of the current study is its nationwide population-based design. The large-scale database we used provided a sufficient sample size to allow rigorous research to be conducted. However, 
Table 3: Comparison of utilization of chemotherapy, invasive procedure, and advanced diagnostic testing between cancer patients with and without dementia 1 and 3 months prior to death

\begin{tabular}{|c|c|c|c|c|c|c|c|c|c|c|}
\hline & \multicolumn{5}{|c|}{1 month prior to death } & \multicolumn{5}{|c|}{3 months prior to death } \\
\hline & \multicolumn{2}{|c|}{$\begin{array}{l}\text { Dementia } \\
(N=7111)\end{array}$} & \multicolumn{2}{|c|}{$\begin{array}{c}\text { No dementia } \\
(N=\mathbf{2 8 4 4 4})\end{array}$} & \multirow[b]{2}{*}{$\begin{array}{c}\text { Adjusted OR } \\
(95 \% \mathrm{CI}) \dagger\end{array}$} & \multicolumn{2}{|c|}{$\begin{array}{l}\text { Dementia } \\
(N=6015)\end{array}$} & \multicolumn{2}{|c|}{$\begin{array}{c}\text { No dementia } \\
(N=\mathbf{2 4 0 6 0 )}\end{array}$} & \multirow[b]{2}{*}{$\begin{array}{c}\text { Adjusted OR } \\
(95 \% \text { CI }) \dagger\end{array}$} \\
\hline & $\mathbf{N}$ & $\%$ & $\mathbf{N}$ & $\%$ & & $\mathbf{N}$ & $\%$ & $\mathbf{N}$ & $\%$ & \\
\hline Chemotherapy & 762 & 10.7 & 4648 & 16.3 & $\begin{array}{c}0.60 \\
(0.55-0.66)^{* * *}\end{array}$ & 1104 & 18.4 & 7066 & 29.4 & $\begin{array}{c}0.51 \\
(0.47-0.55)^{* * *}\end{array}$ \\
\hline \multicolumn{11}{|l|}{ Invasive procedure } \\
\hline $\begin{array}{l}\text { Cardiopulmonary } \\
\text { resuscitation }\end{array}$ & 974 & 13.7 & 2982 & 10.5 & $\begin{array}{c}1.32 \\
(1.22-1.43)^{* * *}\end{array}$ & 879 & 14.6 & 2647 & 11.0 & $\begin{array}{c}1.35 \\
(1.24-1.47)^{* * *}\end{array}$ \\
\hline Endotracheal intubation & 2044 & 28.7 & 6833 & 24.0 & $\begin{array}{c}1.27 \\
(1.19-1.34)^{* * *}\end{array}$ & 1988 & 33.1 & 6423 & 26.7 & $\begin{array}{c}1.34 \\
(1.26-1.42)^{* * *}\end{array}$ \\
\hline Mechanical ventilation & 3196 & 44.9 & 10317 & 36.3 & $\begin{array}{c}1.45 \\
(1.37-1.53)^{* * *}\end{array}$ & 2886 & 48.0 & 9194 & 38.2 & $\begin{array}{c}1.49 \\
(1.40-1.50)^{* * *}\end{array}$ \\
\hline Urinary catheterization & 4558 & 64.1 & 16731 & 58.8 & $\begin{array}{c}1.24 \\
(1.18-1.30)^{* * *}\end{array}$ & 4154 & 69.1 & 15116 & 62.8 & $\begin{array}{c}1.30 \\
(1.22-1.38)^{* * *}\end{array}$ \\
\hline Feeding tube & 5679 & 79.9 & 19192 & 67.5 & $\begin{array}{c}1.88 \\
(1.76-2.00)^{* * *}\end{array}$ & 4921 & 81.8 & 16642 & 69.2 & $\begin{array}{c}1.96 \\
(1.82-2.10)^{* * *}\end{array}$ \\
\hline \multicolumn{11}{|l|}{ Advanced diagnostic testing } \\
\hline CT/MRI/Sonography & 4263 & 60.0 & 17985 & 63.2 & $\begin{array}{c}0.87 \\
(0.82-0.92)^{* * *}\end{array}$ & 4696 & 78.1 & 19572 & 81.4 & $\begin{array}{c}0.78 \\
(0.72-0.83)^{* * *}\end{array}$ \\
\hline Panendoscopy & 924 & 13.0 & 3862 & 13.6 & $\begin{array}{c}0.93 \\
(0.86-1.00)\end{array}$ & 1215 & 20.2 & 4995 & 20.8 & $\begin{array}{c}0.91 \\
(0.85-0.90)^{* *}\end{array}$ \\
\hline Colonoscopy & 198 & 2.8 & 683 & 2.4 & $\begin{array}{c}1.15 \\
(0.98-1.35)\end{array}$ & 242 & 4.0 & 934 & 3.9 & $\begin{array}{c}1.00 \\
(0.87-1.16)\end{array}$ \\
\hline Bone scan & 371 & 5.2 & 2148 & 7.6 & $\begin{array}{c}0.69 \\
(0.61-0.77)^{* * *}\end{array}$ & 608 & 10.1 & 3619 & 15.0 & $\begin{array}{c}0.62 \\
(0.56-0.68)^{* * *}\end{array}$ \\
\hline PET scan & 20 & 0.3 & 105 & 0.4 & $\begin{array}{c}0.80 \\
(0.49-1.30)\end{array}$ & 28 & 0.5 & 186 & 0.8 & $\begin{array}{c}0.61 \\
(0.40-0.91)^{*}\end{array}$ \\
\hline
\end{tabular}

$\dagger$ Multiple logistic regression with adjusted for age, sex, primary malignancy, Charlson comorbidity index, year of death, and insurance premium.

$* \mathrm{p}<0.05, * *_{\mathrm{p}}<0.01, * * *_{\mathrm{p}}<0.0001$

this study also suffers from some limitations. First, the prevalence of dementia was lower than that reported in the literature [33]. The low prevalence that we observed may be related to the stricter screening criteria we adopted. To be included in this research, patients had to possess a catastrophic illness certification for dementia or have been diagnosed with dementia by a neurologist or a psychiatrist. Patients who were diagnosed with dementia by other specialists were not included in this study. However, we believe that using stricter inclusion criteria ensured the accuracy of the dementia diagnoses. Second, the severity of dementia may affect the decision making. However, the claims data does not report the severity of dementia and the patients' level of functioning. Therefore, we do not know if our results are generalizable to all patients with dementia, and further studies may be necessary to explore this issue. Third, evidence obtained in cohort studies can be biased by unmeasured or unknown confounders. Although we attempted to control for some potential confounding factors by including suitable matching and covariates adjustment, other confounders may still exist.
In summary, this study revealed that patients with both cancer and dementia were more likely to be cared for in the ICU and to receive invasive procedures, but less likely to receive chemotherapy, palliative care, or advanced diagnostic testing than those without dementia. Further investigation to gain a better understanding of the decision-making process for patients with dementia and cancer is required.

\section{MATERIALS AND METHODS}

\section{Data sources}

In 1995, Taiwan instituted the NHI program, which is a single-payer program administered by the government. NHI covers approximately $99 \%$ of the Taiwanese population and includes contracts with $97 \%$ of the hospitals and clinics in Taiwan. We used the NHI Research Database containing NHI claims data from 2000 to 2012 obtained from the National Health Research Institute (NHRI). This data includes registries and claims from contracted health-care facilities. After 
receiving NHRI approval, we obtained medical records from the Catastrophic Illness Patient Database and the registry of beneficiaries, as well as ambulatory claims and inpatient care claims. Concerning data security and the patient's privacy, personal identification was encrypted before releasing it from the database. Patient diagnoses were determined using the International Classification of Disease, 9th Revision, Clinical Modification (ICD-9-CM). This study was approved by the Institutional Review Board of the Tzu Chi Medical Center.

\section{Study population}

We used the Catastrophic Illness Patient Database to identify a study cohort that included patients who had been newly diagnosed with one of the six most common cancers in Taiwan between 2000 and 2012. Specifically, these were colorectal (ICD-9 codes 153-154), liver (ICD-9 code 155), lung (ICD-9 code 162), breast (ICD-9 code 174), oral (ICD-9 codes 140-141, 143-146, 148-149), and prostate cancers (ICD-9 code 185). Patients were excluded from the study if they 1) had more than one cancer diagnosis, 2) were younger than 20 years old, 3) remained alive during the follow-up period, 4) had history of traffic accident before death, or 5) did not receive inpatient care within the final month prior death.

To specify a dementia group, we identified patients with cancer from the study cohort who had also been assigned an ICD-9 diagnostic code for dementia during the study period (i.e., ICD-9 codes 290.0-290.4 and 331.0). The ICD-9 dementia codes had to be 1) shown on a catastrophic illness certification for dementia, 2) assigned by a neurologist or a psychiatrist during a hospital stay, or 3) assigned by a neurologist or a psychiatrist in an outpatient clinic at least twice in the same year.

We then matched four patients with cancer but without a dementia diagnosis with each patient with cancer who had a dementia diagnosis. Patients were matched by age, gender, type of primary cancer, year of death, and length of time between cancer diagnosis and death (Figure 1). To account for the possible impact of disease progression on decisions pertaining to end-of-life care, we analyzed patients matched for the length of time between cancer diagnosis and death, comparing variables among those dying within 1 month of diagnosis and also among those dying within 3 months of diagnosis.

\section{Research variables}

We used medical records to identify the comorbid conditions each patient had during the year prior to death, allowing calculation of the Charlson comorbidity index [34]. Demographic and clinical characteristics included age, gender, type of primary cancer, year of death, and length of time between cancer diagnosis and death. We also evaluated the socioeconomic status of patients using income-related insurance premiums as a proxy for income. Specifically, insurance premium was classified into four categories: $\geq 25,001$ New Taiwan dollars (NTD), 15,84125,000 NTD, 1-15,840 NTD, or financially dependent (such as the unemployed, students, children, and elderly persons with no salary).

\section{Study outcomes}

In this study, we investigated how dementia influenced the medical care, such as aggressive treatments or invasive procedures, which a patient with cancer received during the final 1 and 3 months of their lives. More specifically, for patients with and without dementia, we compared 1) length of hospital stay, intensive care unit (ICU) stay, and hospice care; 2) anticancer chemotherapy; 3 ) invasive procedures, including CPR, endotracheal intubation, mechanical ventilation, urinary catheterization, and feeding tubes; and 4) advanced diagnostic tests, including CT or MRI or sonography, panendoscopy, colonoscopy, bone scans, and positron emission tomography (PET) scans.

\section{Statistical analysis}

Continuous variables were analyzed using independent Student's t-tests and a multiple logistic regression model was used to determine whether a dementia diagnosis influenced the likelihood of utilization of types of care, invasive procedures, and advanced diagnostic testing. Corrections were made to account for age, gender, type of primary cancer, Charlson comorbidity index, year of death, length of time between cancer diagnosis and death, and insurance premiums (Table 1). Results are reported as adjusted odd ratios (ORs) with 95\% confidence intervals (CIs). A value of $\mathrm{p}<0.05$ is considered statistically significant. All statistical analyses were performed using SAS 9.4 (SAS Institute, Inc., Cary, NC).

\section{Abbreviations}

OR: odds ratio, CI: confidence interval, ICU: intensive care unit, CPR: cardiopulmonary resuscitation, CT: computed tomography, MRI: magnetic resonance imaging, PET: positron emission tomography, NHI: National Health Insurance, NHRI: National Health Research Institute, NTD: New Taiwan dollars.

\section{Author contributions}

Study conception and design: Huei-Kai Huang and Ying-Wei Wang; acquisition of data: Chia-Jung Hsieh; analysis and interpretation of data: Huei-Kai Huang, Jyh-Gang Hsieh, Chia-Jung Hsieh, and Ying-Wei Wang; drafting of manuscript: Huei-Kai Huang and Ying-Wei Wang; critical revision: Huei-Kai Huang, Jyh-Gang Hsieh, Chia-Jung Hsieh and Ying-Wei Wang. 


\section{ACKNOWLEDGMENTS}

The authors are grateful to Dr. Tang-Yuan Chu for the provision of research database.

\section{CONFLICTS OF INTEREST}

The authors declare that they do not have any conflicts of interest.

\section{FUNDING}

This study was supported by Tzu Chi University, Taiwan (610400353-02).

\section{REFERENCES}

1. Ho TH, Barbera L, Saskin R, Lu H, Neville BA, Earle $\mathrm{CC}$. Trends in the aggressiveness of end-of-life cancer care in the universal health care system of Ontario, Canada. J Clin Oncol. 2011; 29: 1587-91. doi: 10.1200/ jco.2010.31.9897.

2. Ministry of Health and Welfare. 2015 statistics of causes of death Taiwan: Ministry of Health and Welfare; 2015 [cited 2017 Jan. 10]. Available from: http://www.mohw.gov.tw/ CHT/DOS/Statistic.aspx?f_list_no $=474 \&$ fod_list_no $=3443$.

3. Earle CC, Landrum MB, Souza JM, Neville BA, Weeks JC, Ayanian JZ. Aggressiveness of cancer care near the end of life: is it a quality-of-care issue? J Clin Oncol. 2008; 26: 3860-6. doi: 10.1200/jco.2007.15.8253.

4. Wright AA, Zhang B, Keating NL, Weeks JC, Prigerson HG. Associations between palliative chemotherapy and adult cancer patients' end of life care and place of death: prospective cohort study. BMJ. 2014; 348: g1219. doi: 10.1136/bmj.g1219.

5. Temel JS, McCannon J, Greer JA, Jackson VA, Ostler P, Pirl WF, Lynch TJ, Billings JA. Aggressiveness of care in a prospective cohort of patients with advanced NSCLC. Cancer. 2008; 113: 826-33. doi: 10.1002/cncr.23620.

6. Morin L, Beaussant Y, Aubry R, Fastbom J, Johnell K. Aggressiveness of end-of-life care for hospitalized individuals with cancer with and without dementia: a nationwide matched-cohort study in France. J Am Geriatr Soc. 2016; 64: 1851-7. doi: 10.1111/jgs. 14363.

7. Earle CC, Neville BA, Landrum MB, Ayanian JZ, Block $\mathrm{SD}$, Weeks JC. Trends in the aggressiveness of cancer care near the end of life. J Clin Oncol. 2004; 22: 315-21. doi: 10.1200/jco.2004.08.136.

8. Earle CC, Park ER, Lai B, Weeks JC, Ayanian JZ, Block S. Identifying potential indicators of the quality of end-of-life cancer care from administrative data. J Clin Oncol. 2003; 21: 1133-8. doi: 10.1200/jco.2003.03.059.

9. Haller IV, Gessert CE. Utilization of medical services at the end of life in older adults with cognitive impairment: focus on outliers. J Palliat Med. 2007; 10: 400-7. doi: 10.1089/ jpm.2006.0129.

10. Hinkka H, Kosunen E, Lammi EK, Metsanoja R, Puustelli A, Kellokumpu-Lehtinen P. Decision making in terminal care: a survey of finnish doctors' treatment decisions in endof-life scenarios involving a terminal cancer and a terminal dementia patient. Palliat Med. 2002; 16: 195-204. doi: 10.1191/0269216302pm510oa.

11. Goodman C, Froggatt K, Amador S, Mathie E, Mayrhofer A. End of life care interventions for people with dementia in care homes: addressing uncertainty within a framework for service delivery and evaluation. BMC Palliat Care. 2015; 14: 42. doi: 10.1186/s12904-015-0040-0.

12. Afzal N, Buhagiar K, Flood J, Cosgrave M. Quality of end-of-life care for dementia patients during acute hospital admission: a retrospective study in Ireland. Gen Hosp Psychiatry. 2010; 32: 141-6. doi: 10.1016/j. genhosppsych.2009.10.003.

13. Richardson SS, Sullivan G, Hill A, Yu W. Use of aggressive medical treatments near the end of life: differences between patients with and without dementia. Health Serv Res. 2007; 42: 183-200. doi: 10.1111/j.1475-6773.2006.00608.x.

14. Tschirhart EC, Du Q, Kelley AS. Factors influencing the use of intensive procedures at the end of life. J Am Geriatr Soc. 2014; 62: 2088-94. doi: 10.1111/jgs.13104.

15. Ferri CP, Prince M, Brayne C, Brodaty H, Fratiglioni L, Ganguli M, Hall K, Hasegawa K, Hendrie H, Huang Y, Jorm A, Mathers C, Menezes PR, et al. Global prevalence of dementia: a Delphi consensus study. Lancet. 2005; 366: 2112-7. doi: 10.1016/s0140-6736(05)67889-0.

16. Yancik R, Ries LA. Cancer in older persons: an international issue in an aging world. Semin Oncol. 2004; 31: 128-36.

17. Puts MT, Tapscott B, Fitch M, Howell D, Monette J, WanChow-Wah D, Krzyzanowska M, Leighl NB, Springall E, Alibhai SM. A systematic review of factors influencing older adults' decision to accept or decline cancer treatment. Cancer Treat Rev. 2015; 41: 197-215. doi: 10.1016/j. ctrv.2014.12.010.

18. Pang A, Ho S, Lee SC. Cancer physicians' attitude towards treatment of the elderly cancer patient in a developed Asian country. BMC Geriatr. 2013; 13: 35. doi: 10.1186/1471-2318-13-35.

19. Blackhall LJ, Murphy ST, Frank G, Michel V, Azen S. Ethnicity and attitudes toward patient autonomy. JAMA. 1995; 274: 820-5. doi: 10.1001/jama.274.10.820.

20. Phipps E, True G, Harris D, Chong U, Tester W, Chavin SI, Braitman LE. Approaching the end of life: attitudes, preferences, and behaviors of African-American and white patients and their family caregivers. J Clin Oncol. 2003; 21: 549-54. doi: 10.1200/JCO.2003.12.080.

21. Tang ST, Liu TW, Lai MS, Liu LN, Chen CH. Concordance of preferences for end-of-life care between terminally ill cancer patients and their family caregivers in Taiwan. $\mathrm{J}$ 
Pain Symptom Manage. 2005; 30: 510-8. doi: 10.1016/j. jpainsymman.2005.05.019.

22. Maly RC, Umezawa Y, Ratliff CT, Leake B. Racial/ ethnic group differences in treatment decision-making and treatment received among older breast carcinoma patients. Cancer. 2006; 106: 957-65. doi: 10.1002/cncr.21680.

23. Liu TW, Chang WC, Wang HM, Chen JS, Koong SL, Hsiao SC, Tang ST. Use of chemotherapy at the end of life among Taiwanese cancer decedents, 2001-2006. Acta Oncol. 2012; 51: 505-11. doi: 10.3109/0284186x.2011.653440.

24. Ayalon L, Bachner YG, Dwolatzky T, Heinik J. Preferences for end-of-life treatment: concordance between older adults with dementia or mild cognitive impairment and their spouses. Int Psychogeriatr. 2012; 24: 1798-804. doi: 10.1017/s 1041610212000877.

25. Chen CL, Wang YW. The initiation of end of life care communication-analysis of Taiwan promotional films for hospice palliative care. Taiwan J Hosp Palliat Care. 2013; 18: 142-54. doi: 10.6537/tjhpc.2013.18(2).2.

26. Tang ST, Liu TW, Tsai CM, Wang CH, Chang GC, Liu LN. Patient awareness of prognosis, patient-family caregiver congruence on the preferred place of death, and caregiving burden of families contribute to the quality of life for terminally ill cancer patients in Taiwan. Psychooncology. 2008; 17: 1202-9. doi: 10.1002/pon.1343.

27. Wang SC, Chang CJ, Fan SY, Wang YW, Chang SC, Sung HC. Development of an advance care planning booklet in Taiwan. Tzu Chi Med J. 27: 170-4. doi: 10.1016/j. tcmj.2015.07.003.
28. Lo YT, Wang JJ, Liu LF, Wang CN. Prevalence and related factors of do-not-resuscitate directives among nursing home residents in Taiwan. J Am Med Dir Assoc. 2010; 11: 43642. doi: 10.1016/j.jamda.2009.10.006.

29. Huang QS. A review on problems of China's hospice care and analysis of possible solutions. Chin Med J (Engl). 2015; 128: 279-81. doi: 10.4103/0366-6999.149241.

30. Hsieh JG, Wang YW. Promoting advance care planning in Taiwan - a practical approach to Chinese culture. BMJ Support Palliat Care. 2011; 1: 79. doi: 10.1136/ bmjspcare-2011-000053.47.

31. Wu TY, Majeed A, Kuo KN. An overview of the healthcare system in Taiwan. London J Prim Care (Abingdon). 2010; 3: 115-9. doi: 10.1080/17571472.2010.11493315.

32. National Health Insurance Administration. Patients with catastrophic illnesses or rare diseases Taiwan: National Health Insurance Administration; 2016 [cited 2017 Jan. 10]. Available from: http://www.nhi.gov.tw/English/ webdata/webdata.aspx?maleu=11\&maleu_id=596\&WD_ ID $=596 \&$ webdata_id=3180.

33. Miesfeldt S, Murray K, Lucas L, Chang CH, Goodman $\mathrm{D}$, Morden NE. Association of age, gender, and race with intensity of end-of-life care for Medicare beneficiaries with cancer. J Palliat Med. 2012; 15: 548-54. doi: 10.1089/ jpm.2011.0310.

34. Charlson ME, Pompei P, Ales KL, MacKenzie CR. A new method of classifying prognostic comorbidity in longitudinal studies: development and validation. J Chronic Dis. 1987; 40: 373-83. doi: 10.1016/0021-9681(87)90171-8. 